\section{Evaluación de la aplicación del Teatro Foro en el aula como herramienta de aprendizaje en el Grado de Trabajo Social.}

\author{
Evaluation of the application of Forum Theater in the classroom as a learning \\ tool in the Degree in Social Work.
}

Cecilia Serrano-Martínez'

\section{Resumen}

El presente estudio pretende conocer las visiones del estudiantado universitario de Trabajo Social, acerca de su experiencia, emociones vivenciadas y aprendizajes alcanzados tras la aplicación de la herramienta Teatro Foro en el aula durante el curso 2017-2018, en la Universidad de La Rioja. Esta técnica se ha utilizado con el fin pedagógico de aprender a resolver una situación de conflicto que podemos encontrar en la práctica profesional de Trabajo Social, a partir de una escena teatralizada (alumnado de cuarto curso), y por medio de las aportaciones del foro presente (alumnado de segundo curso). Esta dinámica tiene una novedad adicional ya que, la creación e interpretación del caso, ha sido realizada por estudiantado de cuarto curso de Trabajo Social. Este aspecto dota de un carácter intergrupal, que enriquece la visión del contenido a tratar, así como la posterior colaboración entre compañeros/as de diferentes cursos académicos. El Teatro Foro fomenta el aprendizaje activo, promueve el trabajo cooperativo y sirve para comprender la magnitud de las diversas realidades sociales a las que tratamos de dar respuesta desde el Trabajo Social. Algunos de los hallazgos más interesantes son: la importancia de las emociones en el aula, el trabajo intergrupal como recurso de aprendizaje, y las relaciones horizontales como favorecedoras de un clima de trabajo adecuado para el desarrollo de la motivación y la creatividad.

Palabras clave: Trabajo Social, educación superior, teatro foro, innovación docente, aprendizaje experiencial.

\section{Abstract}

The present study aims to know the visions of Social Work university students about their experience, emotions experienced and learning achieved, after the application of the Forum Theatre tool in the classroom during the course 2017-2018 in the University of La Rioja. This technique has been used for the pedagogical purpose of learning to solve a conflict situation that can be found in the professional practice of Social Work, from a theatricalized (fourth-year students) scene and through the contributions of the forum (second-year students). This dynamic has an additional innovation, due to the creation and interpretation of the case has been carried out by the fourth degree of Social Work classmates. This aspect gives an intergroup character, which enriches the vision of the content to be treated, as well as the subsequent collaboration between classmates from different academic courses. The Forum Theatre fosters active learning, promotes cooperative work and serves to understand the magnitude of the diverse social realities to which we try to respond from Social Work. Some of the most interesting discovers are the importance of emotions in the classroom, intergroup work as a learning resource, and horizontal relationships creates an adequate work environment for the development of motivation and creativity.

Keywords: Social Work, higher education, theatre forum, teaching innovation, experiential learning.

Serrano-Martínez, C. (2022). Evaluación de la aplicación del Teatro Foro en el aula como herramienta de aprendizaje en el Grado de Trabajo Social. Itinerarios de Trabajo Social, 2, 27-34.

https://doi.org/10.1344/its.i2.32547
ACEPTADO: 02/11/2021 PUBLICADO: 21/01/2022

${ }^{1}$ Área de Trabajo Social y Servicios Sociales. Departamento de Derecho. Universidad de La Rioja.

$\square$ Cecilia Serrano Martínez. Área de Trabajo Social y Servicios Sociales. Departamento de Derecho. Universidad de La Rioja. C/ La Cigüeña, 60, 26.006, Logroño cecilia.serrano@unirioja.es 


\section{Introducción}

La universidad, dentro del modelo que postula la Declaración de Bolonia de 1999, ha de afrontar nuevos enfoques educativos y recursos didácticos (Guerra et al., 2010; Zabalza, 2006) que posicionen a su profesorado y a las herramientas pedagógicas utilizadas como modelo de un aprendizaje participativo y eficaz. Del mismo modo, el contexto universitario ha de ofrecer respuestas a las actuales necesidades y demandas del alumnado, en cuanto a las diferentes habilidades sociales, recursos y metodologías asociadas a cada profesión. Estudios recientes han demostrado el valor de aplicar esta herramienta en la Educación Superior, en tanto que es un recurso metodológico que logra dar respuesta a varios de los objetivos incluidos en asignaturas de diferentes grados universitarios (García Gómez y de Vicente Hernando, 2020; Maldonado Avendaño y Mosquera Téllez, 2020; Sainz de la Maza Alday, 2021).

Dentro de estas estrategias pedagógicas, el Teatro Foro tiene la particularidad de que permite que el público (foro) intervenga en la obra, realice un debate activo y se implique para modificar las situaciones presentadas, por lo que facilita el aprendizaje dialógico y colaborativo y es un "instrumento eficaz para la comprensión y la búsqueda de soluciones a problemas sociales e intersubjetivos" (Boal, 2004, p. 28). Este es un aspecto clave para el desarrollo y aprendizaje de contenidos académicos y prácticos propios de las profesiones en las que se interviene directamente con personas, como es el caso del Trabajo Social.

Concretamente, para la adaptación del Grado de Trabajo Social al Espacio Europeo de Educación Superior, se realizaron una serie de estudios que dieron lugar al Libro Blanco de Trabajo Social (Vázquez, 2004), en el que se definen las competencias y funciones de los/las trabajadores/as sociales, entre las que aparecen: función preventiva, atención directa, planificación, coordinación, inserción social, entre otras (Ballestero et al., 2013). La aplicación del Teatro Foro en el aula sirve para analizar un caso social y llevar a cabo un diagnóstico e intervención; aspectos que se incluyen como elementos propios de la función preventiva, de atención directa y planificación.

Este recurso teatral facilita la reflexión y la exploración de diversas situaciones que pueden acontecer en la vida real, en la práctica profesional, por lo que desempeña un papel importante en la práctica educativa; ya que se sitúa como una herramienta exploratoria de la naturaleza humana (Motos, 2001). Las propuestas de interpretación y análisis incluidas en el Teatro Foro han de estar relacionadas con situaciones de opresión, las cuales se "dan siempre que alguien utiliza el poder que le da fuerza o su estatuto social, económico, o incluso cultural, para reducir a alguien a la pasividad, sumisión, a la condición de objeto" (Abellán, 2001, p. 188). El uso del Teatro Foro se realiza como una táctica en sí misma, es decir, es un instrumento que va más allá de lo estético, que muestra y seguidamente lleva a cabo una praxis desde el grupo (Alvarado y Álvarez, 2016), desde la colectividad en el aula. Este diálogo de saberes nos ayuda a integrar el conocimiento científico, el cual podría ser aplicado directamente a la realidad (Cibati, 2016). El resultado obtenido a partir de la aplicación del Teatro Foro se vincula con un conocimiento instrumental, interactivo y crítico (Park, 1992). En primer lugar, es un conocimiento instrumental ya que integra el conocimiento académico y el situado, a través de la problemática planteada en el aula. En segundo lugar, se trata de un conocimiento interactivo en tanto que, en la construcción del caso a interpretar, el estudiantado basa sus decisiones en el diálogo y conocimiento mutuo de las personas que lo integran. En tercer lugar, es un conocimiento crítico porque es el foro el que valida o rechaza determinadas soluciones vinculadas con la problemática representada; a la vez que reflexionan conjuntamente sobre el conflicto en sí mismo. Esto es así debido a que las personas que forman el foro pasan a construir la propia acción dramatizada y, además, pueden aprovechar ese proceso de construcción para realizar una evaluación de las dificultades y alternativas de cara a preparar futuras acciones (Boal, 1980).

El Teatro Foro se posiciona como un método de enseñanza innovador, cuyas herramientas pedagógicas están centradas en el aprendizaje con el alumnado. Esta afirmación está en armonía con las demandas esperadas de la universidad del siglo XXI, por parte de distintos organismos internacionales, en tanto que ésta ha de formar a los/las estudiantes como ciudadanos con capacidad crítica (Santos Rego et al., 2017) y con un conocimiento aplicado que redunde en el beneficio de la comunidad; a través de un aprendizaje consciente de su profesión.

\section{Objetivo del estudio y aplicación del Teatro Foro}

El presente estudio tiene como objetivo conocer las visiones del estudiantado universitario de Trabajo Social, acerca de su experiencia, emociones vivenciadas y aprendizajes alcanzados tras la aplicación de la herramienta Teatro Foro en el aula durante el curso 2017-2018, en la Universidad de La Rioja.

El desarrollo de la técnica se ha llevado a cabo a lo largo de tres fases diferenciadas, que son: fase de diseño, fase de acción y fase de evaluación. En primer lugar, la fase de diseño tuvo una duración de un mes y las personas partícipes fueron cuatro estudiantes de cuarto curso que participaron, de manera voluntaria, junto al docente que les explicó la herramienta de Teatro Foro y acompañó en su puesta en práctica. Se realizaron un total cuatro reuniones preparatorias, que versaron sobre los siguientes puntos:

Día 1. Se explica la idea de realizar una actividad de innovación en el aula, puntos clave para su desarrollo y se aporta bibliografía para una mayor 
comprensión. Se comienza a diseñar un caso social y se decide el conflicto principal.

Día 2. Se continúa definiendo el caso a desarrollar, se reparten los personajes y se redacta un guion de acciones a desarrollar, en base a una escucha activa mediante una lluvia de ideas colectiva. Se deciden aspectos clave en la historia, a partir de la improvisación teatral.

Día 3. Representación de la escena. Se trata de un caso de cocreación por lo que, en cada puesta en escena, van apareciendo nuevos elementos que deciden incorporar y/o eliminar. Comienzan a realizar una reflexión acerca de los posibles elementos de análisis que podrían aparecer una vez interpretada la escena frente al foro.

Día 4. Último ensayo, en el que se incluyen tres compañeras de cuarto curso como público. Los personajes ensayan con el objeto que les caracteriza. Se decide que, tras la discusión con el foro, se crearán equipos de trabajo para realizar el diagnóstico social del caso y ellos/as acompañarán en ese proceso; saliendo de su personaje, como compañeros/as del Grado de Trabajo Social.

En esta fase inicial se logran varios objetivos pedagógicos. En primer lugar, dar a conocer una herramienta nueva, que podrían utilizar en su futura profesión. En segundo lugar, generar un espacio de aprendizaje horizontal, entre el profesorado y el estudiantado. En tercer lugar, potenciar un aprendizaje entre pares, ya que los componentes que desarrollarán la escena son los que deciden la problemática a interpretar y diseñan personajes, fases, diálogos, acciones, etc. Este tipo de aprendizaje es muy efectivo, en tanto que se está realizando con el objetivo de impartir el conocimiento adquirido a otras personas, lo cual incluye diferentes maneras de utilizar la información recopilada y transformarla para poder hacerla entendible al foro. En este sentido, cabe señalar que "cuando aprendemos algo, intervienen los tres sistemas (memoria procedimental, episódica y semántica): aprendemos lo que hacemos, dónde lo aprendemos y cómo describir lo que aprendemos" (Biggs, 2005, p. 106). Además de lo anterior, se generan compromisos más allá del currículo académico, que versan en la confianza por la técnica presentada y en el interés por el propio aprendizaje más allá del aula.

En segundo lugar, la fase de acción tuvo lugar el día de la representación del caso a resolver por el foro, elaborado en la fase de diseño. El número de asistentes fue de 42 estudiantes lo cual representa el $88 \%$ del total de estudiantes matriculados en la asignatura "Trabajo Social con individuos y familias", que es a la que se dirigía la actividad. Las personas asistentes no conocían la técnica con anterioridad, por lo que para introducir la dinámica se colocó un cartel en la entrada de la clase que decía: "¡Bienvenidos/as espect-actores y espect-actrices!”. El proceso de la fase de acción tiene una estructura que va desde la presentación de la técnica hasta la discusión. Antes de la representación, el docente que facilitaba la actividad explicó, mediante un texto dramatizado, en qué consistía la dinámica. Los aspectos clave de la actividad que se explicaron fueron:

Nombre de la técnica y fin: analizar un conflicto y reflexionar sobre las alternativas de solución.

Partes de la dinámica: observar el caso familiar, participar en el foro para proponer cambios y elaborar el diagnóstico social.

Filosofía y objetivos: aprender para la acción en la vida real, por medio de un trabajo lúdico que favorece un aprendizaje colaborativo.

Una vez presentados los puntos anteriores, se dio paso a la representación. Tras la puesta en escena, la persona facilitadora comenzó a preguntar al foro y éste participó en la resolución del caso, intercambiándose por personajes y proponiendo alternativas y acciones concretas, haciendo preguntas clave a los/las protagonistas de la historia escenificada, etc. Tras la realización de la parte destinada a la discusión, se dedicó un espacio para hablar de las emociones y sensaciones vivenciadas en la experiencia.

En la última parte de la sesión se elaboró un diagnóstico social por equipos, tal y como se acordó en la fase de diseño. El diagnóstico social es un "elemento fundamental en el proceso metodológico del Trabajo Social, pues las intervenciones y los resultados dependen de una adecuada interpretación y definición conceptual de las causas de las necesidades sociales" (Díaz y Fernández, 2013, p. 432). Esta última parte de la fase de acción permitió la adquisición de elementos clave a aplicar en la disciplina de Trabajo Social. Este aprendizaje se vio reforzado a través de una metodología de trabajo intergrupal; en la que los/ las estudiantes de cuarto tutorizaron y acompañaron en el proceso de elaboración del diagnóstico social a los estudiantes de segundo curso.

En tercer lugar, en la fase de evaluación se realizaron dos cuestionarios, uno para cada uno de los dos roles incluidos en la práctica (dinamizadores y foro). Las respuestas recibidas sirvieron para analizar el valor otorgado a la experiencia, emociones vivenciadas y aprendizajes alcanzados.

\section{Metodología aplicada}

La metodología de análisis se aplicó en la fase de evaluación. Ésta consistió en la realización de dos cuestionarios formados por preguntas abiertas y cerradas, dirigidos uno a cada uno de los roles incluidos en el proceso: alumnado dinamizador (actor y actrices) y alumnado al que se dirigía la acción (foro). Las preguntas cerradas se valoraban en una escala de puntuación del 1 al 10, con el objetivo de tener 
información cuantitativa para su posterior medición. Asimismo, se incluyeron preguntas de escala de Likert para conocer el acuerdo en cuestiones concretas. Las respuestas a las preguntas abiertas ofrecen información relacionada con aspectos más concretos acerca de la técnica y la experiencia evaluada, lo cual facilita la obtención de discursos cualitativos. Se diseñó un modelo de cuestionario para cada rol, siendo el carácter del mismo voluntario y anónimo. En el caso de los dinamizadores las preguntas estaban relacionadas con la valoración de las actividades preparatorias, el proceso en el aula, aspectos a mejorar, emociones vivenciadas y utilidad de la herramienta, entre otros. En el caso de las preguntas dirigidas al foro, se consultó sobre la valoración de la actividad, aprendizajes sobre Trabajo Social con individuos y familias y conocimientos adquiridos con relación a los temas trabajados en la asignatura, aspectos a destacar, emociones, utilidad de la dinámica, etc. El porcentaje de respuesta fue del $100 \%$ en los dinamizadores y del $89 \%$ en el foro. El modo de realizar el cuestionario fue de manera virtual, por medio del dispositivo Google Forms, el cual facilita tanto la recogida de respuestas como el anonimato de la persona entrevistada y poder acceder a la misma en cualquier momento del día; siempre que el alumnado tenga acceso a Internet con un dispositivo que se lo facilite. El uso de las Tecnologías de la Información y la Comunicación (TIC) sirvió para recoger la información y para realizar la posterior retroalimentación de las evaluaciones al conjunto del estudiantado. Las TIC permiten el acceso a información actualizada, el procesamiento y almacenaje de datos de manera inmediata y son canales de comunicación rápida para difundir e intercambiar información (Delgado y Oliver, 2006). Por estas razones, se optó por la realización de un cuestionario de forma virtual, al cual se les animó a responder una vez que finalizó la clase, al margen del horario lectivo.

\section{Resultados}

La evaluación final de la actividad realizada se puede sintetizar en tres apartados principales, que engloban el conjunto de aspectos tratados de manera previa y durante la realización del Teatro Foro. En primer lugar, las reflexiones sobre la actividad plasman la información recogida sobre la evaluación global de la propia acción, tanto por parte de los dinamizadores en sus reuniones y ensayos previos, como por parte del foro en el día de la representación del caso. En segundo lugar, el punto sobre las emociones vivenciadas recoge las experiencias y emociones sentidas en el aula. Durante el proceso de la actividad hubo una gran carga emocional, la cual favoreció el aprendizaje, ya que el propio alumnado sintió que formaba parte de un proceso de resolución de un caso familiar; el cual podría encontrarse en su futura práctica profesional, con las emociones derivadas del éxito o el fracaso de las propuestas realizadas. En tercer lugar, los aprendizajes y la utilidad de la herramienta ponen el punto final evaluativo. Estas últimas reflexiones versan sobre las visiones del estudiantado en relación al Teatro Foro: si lo valoran como un instrumento facilitador del aprendizaje, la posible utilidad que puede tener más allá del aula, etc.

\subsection{Reflexiones sobre la actividad}

Los resultados globales de la realización del Teatro foro son muy positivos, tal y como se extrae de las respuestas recibidas, así como de la retroalimentación posterior realizada por el alumnado en las clases. En cuanto al cuestionario, al preguntarles cómo valoran la actividad, el 54,8\% la puntúan con un 10 y el 32,3\% con 9 puntos. Esto supone que el $87 \%$ de los/las asistentes otorgan la categoría de sobresaliente, frente al $13 \%$ restante que la valora con notable ( 7 y 8 puntos). Entre los aspectos que más valoran aparece el de la participación directa en el caso, es decir, el hecho de posicionarse como parte del conflicto en análisis y permitirles unirse a "la función". Entre algunas de sus afirmaciones consideran que la actividad era "muy dinámica y que nosotros participábamos activamente en ella". En relación con esta idea indican que es una dinámica poco común en las clases y que se debería fomentar su realización, ya que les permite participar de una manera más proactiva y protagonista junto al resto de compañeros/as. Este tipo de metodologías les genera mayor implicación en la actividad propuesta en el aula. La interacción con los personajes es un elemento que también incluyen dentro de esta participación como positivo para ello/as, ya que tenían la posibilidad de ponerse en el lugar de los miembros de la familia y probar diferentes opciones de resolución. Del mismo modo, valoran haber podido aprender de compañeros/as de cuarto curso; de los/las cuales destacan su caracterización e interpretación, a la vez que señalan que: "fueron capaces de representar una situación familiar muy creíble y, por desgracia, común”. Por medio de la participación sienten que sus opiniones son escuchadas, a la vez que les permite intercambiar diversos puntos de vista. De este modo se comparten saberes y perspectivas, que redundan en un beneficio colectivo, ya que las reflexiones son compartidas, comentadas y debatidas por medio del espacio de tiempo destinado al foro.

En relación con lo anterior, también dan importancia al debate posterior, es decir, a la parte de reflexión tras la realización del caso, en la que el foro tiene la palabra. Reconocen que no era sencillo realizar propuestas, ya que éstas modificaban la realidad de la familia representada; lo cual refleja las situaciones familiares con las que podrían intervenir desde su profesión. La parte dirigida a la participación del foro les sirvió para saber cómo actuar en una situación similar, profesionalizando su actitud y modo de aplicar las técnicas de intervención concretas explicadas a lo largo de la asignatura.

Otro elemento que aparece en paralelo es el de la diversión, ya que vinculan el aprendizaje a la parte más lúdica de la propia herramienta. Indican que les ha gustado "ver un caso real que puede suceder en 
muchas familias de una forma divertida, porque nos hemos reído mucho, y es muy dinámica". Se trata de un método de aprendizaje en el que sienten que son partícipes de una especie de juego. De esta manera, jugando, es la forma en la que el individuo, ya sea niño o adulto, es capaz de ser creativo y de usar por completo su personalidad (Winnicott, 1971). Los juegos, al igual que las instituciones, "son extensiones del hombre social y del cuerpo político" (McLuhan, 2001, p. 255) y, además, muchas de las motivaciones del individuo están relacionadas por el espíritu del juego (Knight, 1933). Por ello, es interesante comprobar como una actividad en la que se incluye una metodología lúdica genera una motivación que, en sí misma, es intrínseca al individuo y favorece el desarrollo de su creatividad.

Finalmente, a la hora de puntuar la sesión siguiendo la escala Likert, valorándola entre los siguientes cuatro ítems: "no me ha gustado nada", "indiferente", "ha estado bien" y "me ha gustado mucho"; el 93,5\% de los resultados se incluyen en esta última opción, frente al $6,5 \%$ restante que indican que "ha estado bien". Este aspecto indica el valor concedido a la actividad, en tanto que la mayoría de los/las asistentes lo incluyen en el apartado más positivo.

Además de lo anterior, es interesante conocer la visión de los dinamizadores, tanto en las actividades preparatorias, como en el desarrollo del caso en el aula. En cuanto a las actividades previas, coinciden en la idea de que existió un "buen ambiente, todas las ideas eran escuchadas y fue fácil llegar a un consenso de la situación imaginaria". Añaden que el grupo fue muy flexible a la hora de tomar decisiones y que, sus experiencias personales y los ensayos, les ayudaron a alcanzar los resultados finales. Con relación a lo vivenciado en el aula, el $75 \%$ puntúan la experiencia con un 10 y el $25 \%$ con un 9 . Consideran que hubo una participación activa e interés por parte de sus compañeros de segundo curso, así como una gran creatividad en las soluciones que proponían para resolver el caso planteado. Reconocen sentirse emocionados por la participación y el interés observado durante el Teatro Foro.

\subsection{Emociones vivenciadas}

En una actividad de este tipo, en la que entra en juego la improvisación, la salida a escena y la creatividad aplicada, es vital preguntarse acerca de las emociones vivenciadas durante el proceso en el que acontece la actividad. En este sentido es importante señalar que se fomentó el desarrollo de una creatividad grupal, en la que entran en juego los intercambios entre los compañeros y lo vivenciado en el aula. En este sentido, se trataría de una creatividad entendida como un proceso social amplio, que requiere de un trabajo en equipo (Florida \& Tinagli, 2004). Por lo tanto, no se trata de un bien únicamente individual, sino que es un bien colectivo y relacional. En línea a esta idea de creatividad y grupo, Matusek (1984, p. 199) señala que "la creatividad no está determinada tan solo por los influjos y el sello de la vida personal. Su activación depende también de las circunstancias intrínsecas bajo las que debe realizarse una obra". Siguiendo esta idea, la "obra" sería la propia realización de la escena y la puesta en común de ideas a través del foro. Se trata de un momento creativo y grupal en el que se ponen en juego las diversas emociones que acontecen en un momento concreto de creación e intercambio colectivo.

Las respuestas a la pregunta acerca de cómo se sintieron participando en el Teatro Foro son muy similares. Aparecen, en más de un 70\% de las respuestas, expresiones como: "muy a gusto”, "muy bien” y "muy cómodo/a". Estas percepciones son indicativas de que el clima alcanzado en el aula durante el desarrollo de la actividad fue positivo para la mayoría de los/las asistentes. Al proponerles una serie de emociones que mostrasen cómo se sintieron durante la actividad, el 41,9\% indican sorpresa, el 19,4\% alegría, el $9,7 \%$ enfado. En el resto de porcentajes aparece la tristeza junto a otras emociones que son descritas con diferentes denominaciones como: frustración, pena, diversión, poderoso, movilización, etc. En cuanto a los dinamizadores, el $75 \%$ sintieron alegría y el $25 \%$ impotencia.

Lo interesante de los resultados anteriores es que, en ambos roles, aparece una mezcla de emociones como la alegría y sorpresa, junto al enfado, la frustración, la pena, etc. En ambos casos, la alegría y la sorpresa suponen más de una tercera parte de los porcentajes, frente al resto de emociones indicadas. Esto es indicativo de que la impresión generalizada fue agradable, al margen de estar mezclada con diversas emociones vinculadas con las sensaciones surgidas, fundamentalmente, por la no consecución de la resolución del caso. El hecho de haber alcanzado esta serie de emociones es muy valioso en la consecución de objetivos docentes y de aprendizaje. La alegría anima a jugar, a transcender los límites y a ser más creativos (Frijda, 1986). Por ello, aprender en un contexto en el que priman emociones como la alegría, permite "el desarrollo y el entrenamiento de habilidades físicas (fuerza, resistencia, precisión), de habilidades psicológicas e intelectuales (comprensión de normas, memoria, autocontrol) y de habilidades sociales necesarias para el establecimiento de relaciones de amistad y de apoyo" (Vecina, 2006, p. 11). Estas habilidades pueden ser muy útiles para desarrollar la capacidad de innovación, que es un elemento de gran utilidad en el terreno profesional y que se puede fomentar desde el ámbito universitario, por medio de actividades que la refuercen.

El foro tiene la sensación generalizada de encontrarse bien, cómodos y con ganas de participar y aprender de la actividad. No obstante, tal y como se indicaba anteriormente, han aparecido diversas voces que indican sentir impotencia, rabia y/o frustración al no poder hacer nada por cambiar la situación familiar escenificada. Se tratan de sentimientos entremezclados, tal y como señalan en una ocasión: “me lo he pasado bien, he sentido también rabia e impotencia. Pero la actividad en global es muy positiva". Esta mezcla de sensaciones es común en varios de los/las 
encuestados/as, que dicen haber sentido diversidad de emociones a lo largo de la actividad.

Había tenido numerosos sentimientos: alegría (ya que la exageración de los personajes hacía que fuera divertido), un poco de frustración (al no participar en la obra, aunque fue culpa mía debido a que me acobardé). Pero sobre todo me sentía "poderoso" ya que sentía que podía cambiar esa situación que podía ayudarlos, motivarlos, guiarlos un poco para que se sientan más felices y a la vez puedan transmitir esa energía (...) No digo que sea fácil ese cambio, pero sí posible.

Sobre el comentario anterior, en varias ocasiones afirman sentirse como verdaderos/as trabajadores/as sociales, lo cual es un aspecto para resaltar, ya que una dinámica en el aula ha sido capaz de transmitir la esencia del rol profesional. En una de estas definiciones, dicen: "la verdad que me he sentido como un verdadero trabajador social intentando ayudar en todo momento". El hecho de lograr esta sensación genera un resultado directo en la autoconcepción del propio alumnado sobre su perfil profesional. Éste produce un empoderamiento y/o reafirmación que traspasa la frontera existente entre el rol de estudiante y el rol de profesional; por la que pasan a visualizarse como futuros/as trabajadores/as, como personas que ostentarán unas herramientas concretas de intervención social.

\subsection{Aprendizajes y utilidad de la herramienta} En términos generales, consideran que el Teatro Foro es una herramienta de gran utilidad, ya que: “ver representado un caso y poder probar en directo distintas soluciones posibilita un aprendizaje directo y que marca". Asimismo, indican que se trata de una manera creativa y eficaz de adquirir conocimientos en el aula, debido a que las personas que forman parte del foro son agentes activos de la acción. Este aspecto dota de realismo a la actividad ofrecida y a las posteriores soluciones propuestas. En cuanto a su valoración del aprendizaje relacionado con la materia en la que se llevó a cabo el Teatro Foro, Trabajo Social con individuos y familias, el $78 \%$ del alumnado puntúa su aprendizaje con sobresaliente ( 9 y 10 puntos) y el $22 \%$ restante con notable ( 7 y 8 puntos).

En diversas ocasiones consideran que esta dinámica les ha ayudado a comprender un caso práctico con mayor facilidad. Este tipo de aprendizaje les ayuda a sintetizar conocimientos explicados en el temario de la asignatura. Indican que les sirve para "poder ver y analizar con quien podríamos empezar interviniendo, además de saber qué tipo de preguntas realizar en una entrevista". Este tipo de conocimiento aplicado va en línea con los contenidos trabajados en clase, lo cual refuerza los saberes adquiridos en la parte teórico-práctica de la asignatura. Concretamente, en su valoración sobre la utilidad de la herramienta para comprender un caso familiar, el 58,1\% la puntúan con un 10 , el $25,8 \%$ con un 9 y el $16,1 \%$ con un 8 . Esto significa que las valoraciones son de notable alto-sobresaliente, ya que se sitúan todas por encima de los 8 puntos, y el $83,9 \%$ estaría incluido en la categoría de sobresaliente. Esta herramienta tiene su potencialidad en la acción, ya que es vivencial y se aprende haciendo, aspecto que valoran en más de una ocasión, cuando indican que: "no es lo mismo hacer que aconsejar". Se trata de un aprendizaje experiencial compuesto por cuatro etapas, que son: percibir, pensar, planear y hacer (Junch, 1983, citado en Acosta García, 2007). La percepción estaría relacionada con la recepción de la experiencia concreta (experimentación de la escena y problemática), pensar da lugar a observar de manera reflexiva la situación, planear se refiere a realizar conceptualizaciones abstractas (propuestas de resolución del caso escenificado) y hacer da lugar a la propia experimentación activa (proponer haciendo, formando parte de la escena como personajes y cambiando los roles de alumno por los de actor/actriz). En una ocasión, analizan este aprendizaje experiencial bajo la siguiente definición: "la participación activa que da lugar a un aprendizaje desde la vivencia, reflexión y motivación hacia la acción. Visualizar a la familia como un sistema dinámico". En definitiva, trabajar desde la acción facilita el aprendizaje, puesto que los/las participantes pasan de ser objetos pasivos (espectadores/as) a sujetos activos ("espect-actores" y "espect-actrices") en la actividad realizada.

En el caso de los dinamizadores, éstos consideran que el Teatro Foro es una herramienta "potentísima para el aprendizaje del alumnado a la hora de intervenir". Igualmente, indican que los/las estudiantes pueden observar en primera persona que la metodología del Trabajo Social tiene un proceso y que "hay que avanzar poquito a poco, no es 'ya'. Hay que tener paciencia y disposición”. Por ello, consideran que es muy importante prepararse para adquirir diversas habilidades, así como tener en cuenta otras opiniones y maneras de valorar cada situación y cada caso en el que intervenir. Con relación a estas afirmaciones, Pantoja Ospina et al. (2013) indican que también "existe la posibilidad de que sean los métodos de aprendizaje y los procesos de pensamiento o la forma de relacionarse con las personas del entorno aquellos elementos que faciliten el aprendizaje" (p.99).

El aprendizaje se puede fomentar con la realización de un mayor número de experiencias y de relaciones con el medio, como es el entorno educativo, familiar, de amistades y ocio, entre otros. En relación a esta última idea, la universidad puede sentar bases para la inclusión de métodos que favorezcan la confluencia de diversos estilos de aprendizaje y el establecimiento de modos más horizontales de relación entre profesorado y estudiantado. Una comunicación fluida entre ambos perfiles "favorece la cordialidad y la cohesión, garantizando un buen clima de trabajo y potenciando la creación de lazos afectivos" (Puig Gutiérrez y Morales Lozano, 2015, p. 273). Este tipo de relaciones más cercanas y continuadas ayudan a alcanzar una mejor adaptación del alumno a la 
universidad y, además, es relevante para su bienestar psicológico y social (Tuero et al., 2018). Este tipo de metodologías de carácter participativo y experiencial favorecen la relación y el aprendizaje horizontal, a la vez que refuerzan los conocimientos académicos y las relaciones sociales.

Fruto de la valoración sobre la utilidad de la herramienta y de los aprendizajes alcanzados con la misma, el estudiantado termina proponiendo la realización de actividades similares, ya que consideran que "fue una manera diferente de aprender y una experiencia enriquecedora para nuestra formación". Del mismo modo, los/las alumnos/as de segundo curso valoran especialmente que se realicen actividades intergrupales, en las que tengan cabida estudiantes de cursos superiores, con el objetivo de aprender de los mismos y poder compartir inquietudes y dudas. Este tipo de intercambios facilitarían la relación entre compañeros/as y fomentaría el aprendizaje colaborativo y entre pares.

\section{Conclusiones}

El Teatro Foro puede ser considerado como una herramienta pedagógica que encajaría en los nuevos enfoques educativos por los que aboga la Declaración de Bolonia de 1999 (Guerra et al., 2010; Zabalza, 2006), en tanto que logra dar respuesta a las necesidades y demandas actuales del estudiantado. Éstas están relacionadas con la idea de aprender haciendo, es decir, experimentando prácticas que se asemejen a su práctica profesional. Este objetivo se ha alcanzado de una manera exitosa y, entre algunos resultados a destacar, aparecen: aprendizaje experiencial, profesionalización, motivación y desarrollo de la creatividad. Además de lo anterior, se han logrado diversos objetivos de interés pedagógico, que son: dar a conocer al estudiantado una herramienta alternativa de intervención, generar un espacio de encuentro y reflexión caracterizado por la horizontalidad entre el profesorado y el alumnado, potenciar un aprendizaje entre pares y un aprendizaje experiencial que incluye elementos lúdicos, y fomentar emociones agradables en el aula.

Como se ha podido observar a lo largo del presente estudio, las emociones son necesarias para el desarrollo de la creatividad (Frijda, 1986) y para el entrenamiento de una serie de habilidades físicas, psicológicas y sociales (Vecina, 2006) que favorecen la consecución los objetivos demandados de la Educación Superior del siglo XXI (Santos Rego et al., 2017), ya que se logra mayor capacidad crítica y un aprendizaje consciente de la profesión. El modelo de aprendizaje experiencial que se alcanza por medio del Teatro Foro tiene una gran carga emocional, siendo la alegría y la sorpresa las emociones que más han aparecido en los cuestionarios analizados. Por todo lo anterior, esta herramienta es un método de enseñanza innovador, capaz de transmitir la esencia del rol profesional, además de generar interés y motivación en el aprendizaje y el trabajo en el aula de manera intergrupal y horizontal. Este método de enseñanza fomenta las relaciones entre profesorado y alumnado, lo cual coincide con diversos estudios (Puig Gutiérrez y Morales Lozano, 2015; Tuero et al., 2018) que señalan los efectos positivos que éstas tienen en los resultados académicos y relacionales del estudiantado. Los/las estudiantes universitarios valoran especialmente el hecho de aprender haciendo y experimentando, siendo partícipes de la resolución del caso. Igualmente consideran, como un valor añadido, que se les brinde la posibilidad de compartir conocimiento con compañeros de cursos superiores. Este aprendizaje intergrupal es un elemento motivador en su desarrollo académico, así como la cercanía con el profesorado, lo cual dota de mayor motivación por la tarea. El conjunto de estos elementos favorece la creatividad aplicada del estudiantado en el aula. Por ello, una propuesta metodológica para el desarrollo de actividades didácticas en las que se opte por incluir el Teatro Foro sería la de incorporar los siguientes elementos que favorecen y complementan este recurso:

- La conexión intergrupos: incluir a alumnos/as de otros cursos, preferiblemente superiores, que faciliten el desarrollo de la escena. De este modo, éstos/ as aprenden a utilizar la herramienta y comparten sus saberes con compañeros/as de cursos inferiores.

- La figura del/la profesor/a facilitador/a: docente entendido como un/a posibilitador/a de procesos y compañero/a que guía el proceso de aprendizaje; en contra de ideas relacionadas con un posicionamiento exclusivamente magistral y/o directivo, ya que se podrían generar barreras a la capacidad creativa y de acción del alumnado.

- Espacios finales de trabajo: organizar grupos más pequeños de trabajo que favorezcan la aplicación concreta del caso. En este espacio tendrían lugar las relaciones más cercanas entre compañeros/ as de diferentes cursos, así como la aclaración de dudas específicas vinculadas con el caso expuesto y con la materia trabajada en la asignatura.

Esta acción pedagógica, de manera similar, se ha desarrollado en diversas universidades españolas. Además, la metodología de análisis utilizada coincide con investigaciones que han medido el grado de satisfacción con los aprendizajes obtenidos, tras utilizar el teatro como herramienta docente (Sainz de la Maza Alday, 2021). Asimismo, en este estudio se han obtenido resultados afines a los alcanzados en otras experiencias que utilizan el Teatro Foro en el espacio universitario, en tanto se han desarrollado procesos de aprendizaje colectivo y de interacción que redundan en una comprensión de los hechos (García Gómez y de Vicente Hernando, 2020); fomentando la comunicación y las relaciones centradas en la gestión de los conflictos (Maldonado Avendaño y Mosquera Téllez, 2020). 
Por medio de esta acción en el aula se ha promovido un aprendizaje experiencial, el cual ha impulsado la adquisición de conocimientos, motivación y desarrollo de la creatividad en el alumnado universitario objeto de esta actividad innovadora. El Teatro Foro ha fomentado un aprendizaje consciente y activo, por medio de un trabajo cooperativo y un diálogo intergrupal. Del mismo modo, ha servido para ofrecer al estudiantado una experiencia aplicada que favorece su profesionalización, y un espacio para reflexionar acerca de la magnitud y de las diversas realidades sociales a las que tratamos de dar respuesta desde el Trabajo Social.

\section{Referencias bibliográficas}

Abellán, J. (2001). Boal conta Boal. Institut del Teatre. Acosta García, M. A. (2007). Estudio de variables relacionadas con la escritura de la lengua inglesa en alumnos de primero y segundo año de la Universidad de Ciego Ávila. (Tesis doctoral). Universidad de Granada.

Alvarado Castro, I., y Álvarez Barragán, G. (2016). El Teatro Foro como herramienta entre el diagnóstico y la programación comunitaria. La mirada antropológica y el desarrollo comunitario se encuentran en Taco (Tenerife). En D. Carbonero, E. Raya, N. Caparros y C. Gimeno. (Coords.). Respuestas transdisciplinares en una sociedad global. Aportaciones desde el Trabajo Social, 1-22. Universidad de La Rioja.

Ballestero Izquierdo, A., Viscarret Garro. J. J., y Úriz Pemán, M. J. (2013). Funciones profesionales de los trabajadores sociales en España. Cuadernos de Trabajo Social, 26(1), 127-138. https://doi.org/10.5209/ rev_CUTS.2013.v26.n1.41664

Biggs, J. (2005). Calidad del aprendizaje universitario. Narcea.

Boal, A. (1980). Teatro del Oprimido: teoría y práctica. Nueva Imagen.

Boal, A. (2004). El arco iris del deseo. Alba Editorial.

Cibati, D. (2016). El Teatro-Foro como herramienta de Investigación Acción Participativa: Una mirada desde la perspectiva decolonial. En D. Carbonero, E. Raya, N. Caparros y C. Gimeno, (Coords.). Respuestas transdisciplinares en una sociedad global. Aportaciones desde el Trabajo Social, 1-13. Universidad de La Rioja.

Delgado, A. M., y Oliver, R. (2006). La evaluación continua en un nuevo escenario docente. Revista de Universidad y Sociedad del Conocimiento (RUSC). 3(1), 1-13.

Díaz Herráiz, E., y Fernández De Castro, P. (2013). Conceptualización del diagnóstico en Trabajo Social: necesidades sociales básicas. Cuadernos de Trabajo Social, 26(2), 431-443. https://doi. org/10.5209/rev_CUTS.2013.v26.n2.39550

Florida, R., \& Tinagli, I. (2004). Europe in the Creative Age. Carnegie Mellon: Alfred P. Sloan Foundation and Demos.

Frijda, N. H. (1986). The emotions. Cambridge University Press.

García Gómez, T., y de Vicente Hernando, C. (2020).
El Teatro-Foro como herramienta didáctica para el cambio educativo. Educación XX1, 23(1). https://doi. org/10.5944/educxx1.23347

Guerra, S., Gonzáles, N., y García-Ruiz, R. (2010). Utilización de las TIC por el profesorado universitario como recurso didáctico. Comunicar, 35, 141-148. http://hdl.handle.net/11162/87143

Knight, F. (1933). Risk, uncertainty and profit. Percy Lund, Humphries.

Maldonado Avendaño, J., y Mosquera Téllez, J. (2020). Teatro foro y pedagogía activa como estrategia para la gestión de conflictos y la transformación social. Revista Palobra, 20(2), 134-149. https://doi. org/10.32997/2346-2884-vol.20-num.2-2020-3312

Matusek, P. (1984). La creatividad. Desde una perspectiva psicodinámica. Herder.

McLuhan, M. (2001). Understanding Media. Routledge Classics.

Motos, T. (2001). Creatividad dramática. Meubook.

Pantoja Ospina, M. A., Duque Salazar, L. I., y Correa Meneses, J. S. (2013). Modelos de estilos de aprendizaje: una actualización para su revisión y análisis. Revista Colombiana de Educación, 64,79-105.

Park, P. (1992). Qué es la investigación-acción participativa. Perspectivas teóricas y Metodológicas. En M. C. Salazar, (Coord.), La Investigaciónacción participativa: inicios y desarrollos,135-174. Editorial Popular.

Puig Gutiérrez, M., y Morales Lozano, J. A. (2015). La formación de ciudadanos: conceptualización y desarrollo de la competencia social y cívica. Educación XX1, 18(1), 259-282. https://doi. org/10.5944/educxx1.18.1.12332

Sainz de la Maza Alday, E. (2021). El juego teatral y el Teatro Foro como herramienta pedagógica y de transformación social: hacia un nuevo paradigma educativo. En A.L. González-Hermosilla (Coord.), Reflexiones y propuestas para los desafíos de la educación actual, 106-115. Adaya Press.

Santos Rego, M. A., Jover Olmeda, G., Naval, C., Álvarez Castillo, J. L., Vázquez Verdera, V., y Sotelino Losada, A. (2017). Diseño y validación de un cuestionario sobre práctica docente y actitud del profesorado universitario hacia la innovación (CUPAIN). Educación XX1, 20(2), 39-71. https://doi.org/10.5944/educxx1.19031

Tuero, E., Cervero, A., Esteban, M., y Bernardo, A. (2018). ¿Por qué abandonan los alumnos universitarios? Variables de influencia en el planteamiento y consolidación del abandono. Educación XX1, 21(2),131154. https://doi.org/10.5944/educxx1.20066

Vázquez, O. (Coord.) (2004). Título de Grado en Trabajo Social (Libro blanco). Aneca.

Vecina Jiménez, M.A. (2006). Emociones positivas. Papeles del Psicólogo, 27(1), 9-17.

Winnicott, D. W. (1971). Playing and reality. Tavistock Publications.

Zabalza, M. A. (2006). La convergencia como oportunidad para la mejora de la docencia universitaria. Revista Interuniversitaria de Formación del Profesorado, 20(3), 37-69. 